\title{
USO DO GESTO NO TRANSTORNO AUTISTA: ESTUDO DE CASO ÚNICO
}

\section{Using the gesture in autism: case report}

\author{
Raquel Prestes ${ }^{(1)}$, Ana Carina Tamanaha ${ }^{(2)}$, Jacy Perissinoto ${ }^{(3)}$
}

\section{RESUMO}

Objetivo: analisar a quantidade e qualidade dos gestos produzidos por uma criança com Transtorno Autista. Procedimentos: trata-se de estudo de caso. Foram utilizados critérios para análise quanto à forma, função e tipo de gestos. Resultados: após seis meses de intervenção terapêutica fonoaudiológica foi observado o aumento de gestos com funcionalidade em relação ao período inicial e após um ano, ausência de movimentos aleatórios. Conclusão: verificou-se aumento na qualidade gestual do caso em questão.

DESCRITORES: Transtorno Autista; Gestos; Comunicação

\section{INTRODUÇÃO}

Os gestos são complementos da linguagem, mostrando ser uma valiosa alternativa ou complemento das palavras, especialmente na formação inicial de vocabulário da criança, essa linguagem gestual se desenvolve aos nove ou dez meses. 0 uso dos gestos pode ser mais informativo que a produção e compreensão de palavras em crianças típicas ${ }^{1-3}$.

O Transtorno Autista tem como característica prejuízo na interação social, comunicação e repertório restrito de atividades. O prejuízo na comuni-

(1) Aluna do Curso de Graduação em Fonoaudiologia da Universidade Federal de São Paulo, UNIFESP, São Paulo, SP.

(2) Fonoaudióloga; Professora Convidada da Disciplina dos Distúrbios da Comunicação Humana da Universidade Federal de São Paulo, UNIFESP, São Paulo, SP; Doutora em Distúrbios da Comunicação Humana pela Universidade Federal de São Paulo.

(3) Fonoaudióloga; Professora Associada do Departamento de Fonoaudiologia da Universidade Federal de São Paulo, UNIFESP, São Paulo, SP; Especialista em Linguagem pelo Conselho Federal de Fonoaudiologia; Mestre em Linguística Aplicada pela Pontifícia Universidade Católica de São Paulo; Doutora em Distúrbios da Comunicação Humana pela Universidade Federal de São Paulo.

Apresentado em forma de pôster no 15 Congresso Brasileiro de Fonoaudiologia e $7^{\circ}$ Congresso Internacional de Fonoaudiologia. Local do evento: Gramado- Rio Grande do Sul- Brasil. Data 16 a 20 de outubro de 2007.

Conflito de interesses: inexistente cação afeta habilidades verbais e não verbais ${ }^{3-5}$, o que inclui gestos e expressões faciais. Os indivíduos autistas utilizam gestos, porém não de forma ajustada às diferentes situações sociais ${ }^{6-9}$. As crianças com Transtorno Autista utilizam os gestos para acompanhar e para substituir o discurso, entretanto nunca para complementá-lo, esta utilização limitada dos gestos é um dos sinais de inabilidade de crianças autistas para o estabelecimento funcional e direcional da comunicação e no desenvolvimento de idéias ${ }^{10,11}$.

Em um estudo sobre o desenvolvimento da comunicação, os autores mostraram que os gestos contribuem para o desenvolvimento da linguagem, uma vez que são funcionais para a comunicação, fazendo a transição para linguagem falada ${ }^{12}$. Assim, diferentes pesquisas sugerem que o gesto pode ter valioso papel no diagnóstico, no prognóstico de distúrbios da comunicação, e na intervenção dos mesmos, pois podem ser utilizados na prática clínica para a ampliação da comunicação 10-12.

Com o intuito de estudar a comunicação gestual ao longo de processo terapêutico fonoaudiológico, este estudo objetivou a análise da quantidade e da qualidade dos gestos produzidos por uma criança com Transtorno Autista, como meio de expressão.

\section{APRESENTAÇÃO DO CASO}

W. A, 10 anos e 7 meses, sexo masculino, diagnosticado por equipe multidisciplinar composta 
por neurologista, psicóloga e fonoaudiólogas com Transtorno Autista, segundo os critérios diagnósticos da CID 10 (1998) e DSM IV Tr (2002) e atendido no Laboratório de Investigação Fonoaudiológica - Transtornos Globais do Desenvolvimento do Depto de Fonoaudiologia da UNIFESP em intervenção terapêutica fonoaudiológica direta e indireta.

A criança apresenta limiares auditivos compatíveis com os parâmetros de normalidade. A avaliação neurológica indicou resultados normais com exceção das alterações comportamentais características do transtorno. E a avaliação psicológica indicou presença de retardo mental de grau moderado e inabilidades nas funções adaptativas.

W. é atendido semanalmente, em sessões individuais (intervenção direta) e sua família participa do grupo de orientações aos pais (intervenção indireta) em sessões quinzenais.

No resgate da história de desenvolvimento foram observados prejuízos sociais e de comunicação precoces. Segundo relato da mãe, a criança sempre foi bastante isolada, não tinha interesse por compartilhar atividades e situações com seus interlocutores. Apresentou atraso na aquisição de fala. Produziu as primeiras palavras apenas aos quatro anos, de forma ecolálica e sempre demonstrou dificuldade em compreender e responder aos estímulos sociais e ambientais.

Seu repertório de atividades é bastante restrito. Não se interessa por brinquedos compatíveis com sua idade. Demonstra interesse exacerbado por jogos de futebol e é capaz de narrar trechos de jogos assistidos com extrema exatidão, embora seja incapaz de compreender e discutir regras simples ou temas pertinentes a este tipo de atividade. Em momentos de ansiedade ou de euforia, observa-se movimentos estereotipados (balanceio de mãos).

Segundo relato da família ainda, não houve intercorrência pré, peri ou pós natais ou menção a outros casos na família.

Para o estudo da caracterização dos gestos da criança foram selecionadas, transcritas e analisadas filmagens de três sessões de terapia fonoaudiológica de 45 minutos de duração, em períodos distintos: 1a. sessão de terapia fonoaudiológica (momento I); após seis meses de terapia (momento II) e após um ano (momento III).

Todas as sessões foram gravadas em situações lúdicas com a díade terapeuta-paciente.

Após a transcrição, as pesquisadoras analisaram os gestos quanto à forma, a função e o tipo.
Quanto à Forma considerou-se: quantidade de movimentos corporais, de cabeça ou mãos ${ }^{13}$.

Quanto à Função ${ }^{10-13}$, adotou-se os seguintes critérios:

1) Emblemáticos: gestos aprendidos culturalmente

2) Reguladores: gestos conscientes; ilustradores, aprendidos, que acompanham a fala, enfatizando a palavra ou a frase. São conscientes, mas não tanto quanto os emblemáticos;

3) Adaptatores: atos não-verbais que regulam e mantêm a comunicação entre dois ou mais interlocutores. Eles não pertencem a um código específico, e são de difícil inibição, pois estão na periferia da consciência;

4) Manifestações afetivas: configurações faciais que assinalam estados afetivos, nos momentos em que não conseguem expressar sentimentos verbalmente.

Em relação ao Tipo ${ }^{10}$, considerou-se os seguintes critérios:

a) Substituição: quando o gesto representou um elemento não presente no discurso oral;

b) Complementação: quando o gesto completou o significado da fala;

c) Aleatório: quando não houve relação com o discurso.

Este relato de caso foi aprovado pelo Comitê de Ética em Pesquisa sob número 1926/07.

O registro ocorreu em planilha desenvolvida para esse estudo.

Para análise dos dados foi considerada a comparação da frequência de uso dos gestos quanto à forma, função e tipo ao longo de 12 meses de intervenção terapêutica fonoaudiológica.

\section{RESULTADOS}

De modo geral, houve diminuição significativa dos gestos quanto à forma, mostrando redução das atipias dos movimentos sem função, tanto em relação à movimentação de corpo, quanto de cabeça e mãos (Figura 1).

Observa-se que em relação à Função, os gestos tornaram-se, ao longo do tempo, mais reguladores e adaptativos, e menos emblemáticos (Figura 2).

Quanto ao Tipo houve diminuição expressiva dos gestos aleatórios e indício de acréscimo de tentativas de gestos complementares (Figura 3). 


\section{Quanto a forma}

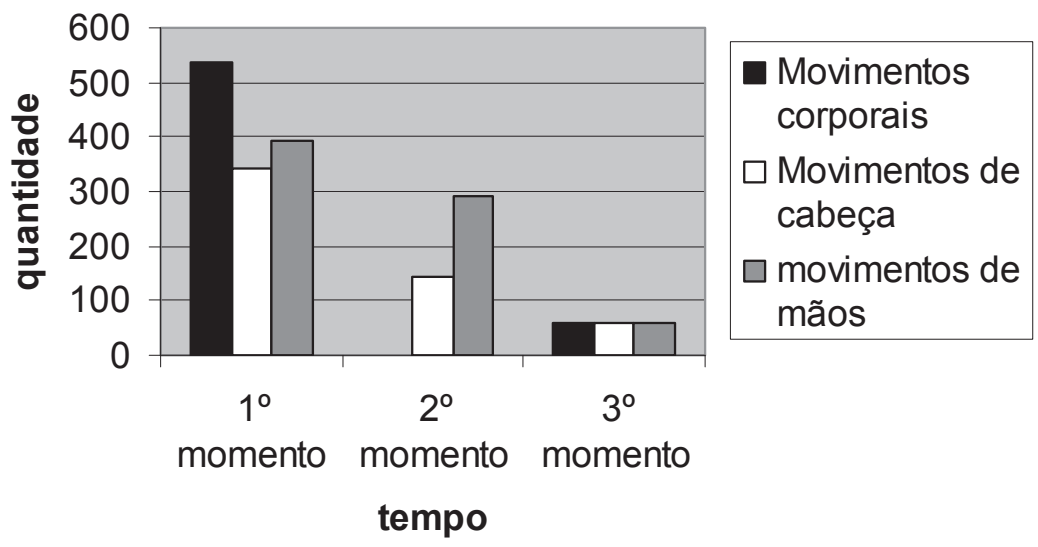

Figura 1 - Frequência de Gestos quanto à Forma nos três momentos analisados

\section{Quanto a função}

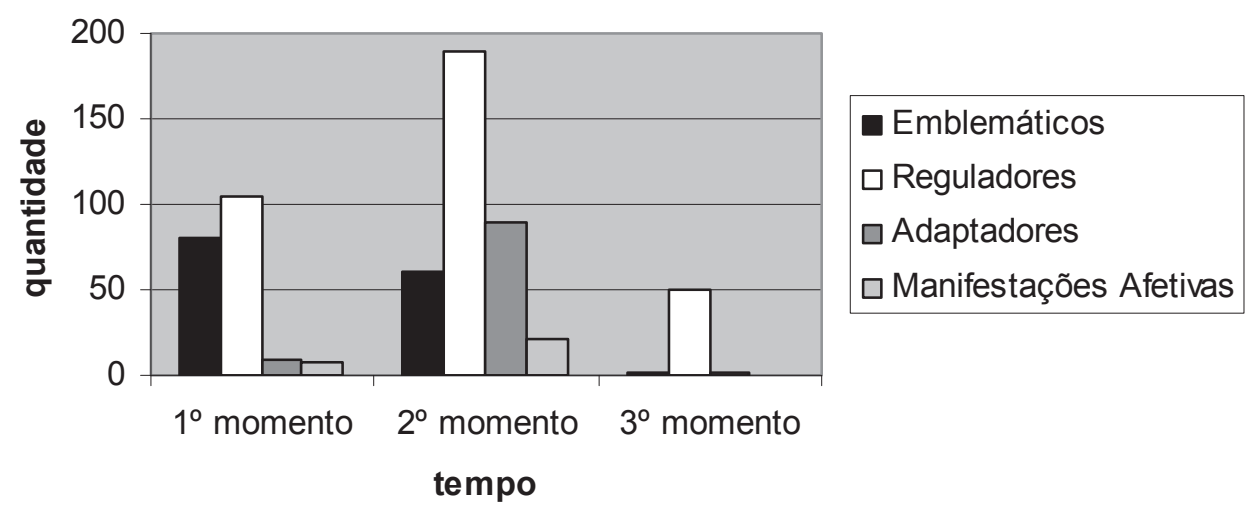

Figura 2 - Frequência de Gestos quanto à Função nos três momentos analisados

Tipos de movimentos

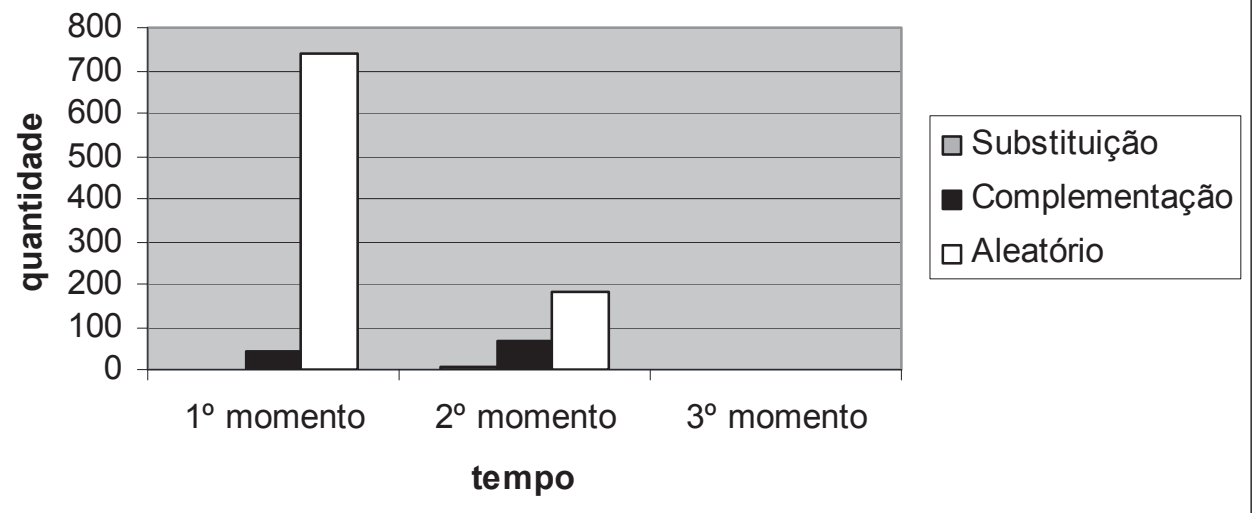

Figura 3 - Frequência de Gestos quanto ao Tipo nos três momentos analisados 


\section{DISCUSSÃO}

Houve um decréscimo expressivo do uso de todas as formas de gestos, ou seja, no inicio da intervenção terapêutica fonoaudiológica registrouse 535 movimentos corporais, 344 de cabeça e 393 de mãos e após 12 meses, foram verificados 50, 60 e 63 movimentos, respectivamente. Esses resultados (Figura 1) mostram que os movimentos excessivos da criança no período inicial estavam vinculados à dificuldade em compartilhar atividades e interesses e aos comportamentos de agitação. Ou seja, os movimentos corporais, de cabeça e de mãos não estavam relacionados ao contexto comunicativo, de modo que não foram reconhecidos, em sua maioria, como formas típicas de apoio de comunicação e sim, como resultado de dificuldade de concentração e atenção. É importante salientar ainda, que muitos destes gestos eram movimentos estereotipados.

Vários estudos têm apontado para a necessidade da observação dos sinais não verbais dentro de contextos comunicativos, pois estes podem ampliar, complementar ou substituir tentativas verbais de comunicação ${ }^{5-10}$.

No entanto, os estudos que buscam caracterizar as inabilidades sociais e de comunicação de indivíduos autistas mostram que estes sujeitos não utilizam gestos como modo alternativo de comunicação, pois a dificuldade em demonstrar a intencionalidade abrange também os sinais não verbais $^{7-14}$.

Esses achados mostram a importância de analisar o uso de gestos não apenas quanto a sua frequência e forma, mas de modo complementar quanto ao seu tipo e especialmente, sua função.

Em relação à análise da função (Figura 2) verificou-se que os gestos emblemáticos, aqueles que são aprendidos culturalmente e são considerados conscientes, diminuíram principalmente no segundo semestre do estudo. Em contrapartida, houve aumento expressivo dos gestos reguladores, especialmente no primeiro semestre. Esses acha- dos devem estar relacionados à eficácia da intervenção terapêutica fonoaudiológica, uma vez que a criança passou a utilizar gestos para regular 0 contexto comunicativo e desta maneira, entende-se que houve maior promoção de situações dialógicas entre terapeuta e paciente.

Nota-se também que houve indício de aumento de gestos com função adaptativa e como manifestação afetiva ao longo do primeiro semestre, mostrando que nos primeiros seis meses de intervenção já foi possível identificar mudanças positivas na criança.

Em relação ao Tipo dos gestos observa-se que a criança apresentou somente um evento de substituição da fala pelo gesto no segundo momento de análise. Quanto à complementação houve aumento expressivo especialmente ao longo do primeiro semestre, ou seja, entre o $1^{\circ}$ e o $2^{\circ}$ momento do estudo (Figura 3). Houve queda notável dos movimentos aleatórios durante todo período do estudo. Esses dados confirmam que a somatória das intervenções direta e indireta foi eficaz em conter as movimentações excessivas da criança e permitir a mesma se engajar em situações comunicativas de forma mais apropriada ${ }^{15-17}$.

Sendo assim, a análise dos gestos tornou-se um aspecto importante no desempenho comunicativo da criança autista, por ser um indicador das inabilidades sociais e de linguagem não verbal. De forma complementar, deve-se lembrar que movimentação excessiva, seja por meio de movimentos estereotipados, seja por comportamentos não adaptativos decorrentes de agitação, ansiedade e desatenção podem mascarar tentativas de comunicação interpessoal e devem portanto ser cuidadosamente assistidas em intervenção terapêutica ${ }^{15-17}$.

\section{CONCLUSÃO}

Foi possível a análise da comunicação gestual ao longo do processo terapêutico fonoaudiológico do caso clínico em questão e mostrar que houve mudança expressiva quanto à forma, função e tipo dos gestos produzidos.

\section{ABSTRACT \\ Purpose: to analyze the quantity and quality of gestures produced by an autistic child. Procedures: case report. Criteria were used for analyzing the form, function and type of gestures. Results: after 6-month therapy, the analyzed subject obtained gestures with functionality above the therapy initiation state. After a year of therapy the subject has not produced more random movements. Conclusion: improvement was verified in gestural quality in this case report.}

KEYWORDS: Autistic Disorder; Gesture; Communication 


\section{REFERÊNCIAS}

1. Papalia DE, Olds SW. Desenvolvimento psicossocial na adolescência. In: Papalia DE, Olds SW. Desenvolvimento humano. 8. ed. Porto Alegre: Artmed; 2006.

2. Andrade SA, Santos DN, Bastos AC, Pedromônico MRM, Almeida Filho N, Barreto ML. Ambiente familiar e desenvolvimento cognitivo infantil: uma abordagem epidemiológica. Rev Saúde Públ. 2005; 39(4):606-11.

3. Mitchell S, Brian J, Zwaigenbaum L, Roberts W, Szatmari P, Smith I, et al. Early language and communication development of infants later diagnosed with autism spectrum disorder. J Develop Behav Pediatr. 2006; 27(Supl2):69-78.

4. American Psychiatric Association. DSM-IV - Manual diagnóstico e estatístico de transtornos mentais. 4. ed. Porto Alegre: Artes Médicas; 1995.

5. Wetherby AM, Prizant BM. Introduction to autism spectrum disorders. In: Whetherby AM, Prizant BM, organizadores. Autism spectrum disorders: a transcortical developmental perspective. Baltimore: Paul Brooks; 2001.p.1-7.

6. Lampreia C. Os enfoques cognitivista e desenvolvimentista no autismo: uma análise preliminar. Psicol Reflex Crít. 2004; 17(1):111-20.

7. Fernandes FDM, Alvejonas-Molini DR, MoratiSousa PF. Perfil funcional da comunicação nos distúrbios do espectro autístico. Rev CEFAC. 2006; 8(1):20-6.

8. Gadia CA, Tuchman R, Rotta NT. Autismo e doenças invasivas de desenvolvimento. J Pediatr. 2004; 80(Supl2):83-94.
9. Melo TM, Saidah SH, Herrera SAL, Hage SVR. Relações entre alterações anatomofuncionais do sistema nervoso central e autismo. Rev CEFAC. 2006; 8(1):36-41.

10. Puglisi M, Mantovan J, Perissinoto J. Caracterização da utilização de gestos por crianças com transtorno autístico durante a narração de histórias. Temas Desenvolv. 2005; 14(80):68-74.

11. Farah LSD, Goldenber M. O autismo entre dois pontos. Rev CEFAC. 2001; 3(1):19-26.

12. Capone NC, McGregorKK. Gesture development: a review for clinical and research practices. J Speech Lang Hear Res. 2004; 47(1):173-88.

13. Wetherby AM. Profiling pragmatic abilities in the emerging language of young children. In: Gallagher TM. Pragmatics of language. Clinical practice issues. San Diego: Singular Publishing Group, Inc; 1991. p.2479-27.

14. Pastorello LM. Contribuições da abordagem funcionalista do desenvolvimento da linguagem: análise clínica de dois casos. In: Fernandes FDM, Pastorello LM, Scheuer $\mathrm{Cl}$, organizador. Fonaudiologia em distúrbios psiquiátricos da infância. São Paulo: Lovise; 1996. p.27-35.

15. Martins MFP. O apoio do verbal e do gestual na elaboração de narrativas pela criança em situações de interação com adulto. [dissertação] São Paulo (SP): Universidade de São Paulo; 2000.

16. Kenn D. The use of non-verbal repair strategies by children with autism. Rev Develop Disabil. 2005; 26(3):243-54.

17. Perissinoto J. Processo de evolução da criança com autismo. In: Perissinoto J. Conhecimentos Essenciais para atender bem a criança com autismo. São José dos Campos: Pulso; 2003.p.23-7.

DOI: 10.1590/S1516-18462009005000032

RECEBIDO EM: 29/06/2008

ACEITO EM: 26/01/2009

Endereço para correspondência:

Raquel Prestes

Rua Barão do Rio Branco, 223 ap. 117

Jundiaí - SP

CEP: 13201-670

E-mail: raquelprestesfono@gmail.com / raquelprestesfono@yahoo.com.br 